\title{
Ultrathin lead bromide perovskite platelets spotted with europium(II) bromide nanodots
}

\author{
Ignacio Rosa-Pardo, ${ }^{a}$ Salvador Pocoví, ${ }^{a}$ Raul Arenal,, ${ }^{b, c, d}$ Raquel E. Galian, ${ }^{* a}$ and Julia Pérez- Prieto*a
}

We describe here the preparation of a novel nanohybrid comprising a two-layer cesium lead bromide nanoplatelet, [CsPbBr 3$] \mathrm{PbBr}_{4} \mathrm{NPL}$, containing europium(II) bromide $\left(\mathrm{EuBr}_{2}\right)$ nanodots, by ultrasound/heating treatment of toluene dispersions of $\mathrm{CsPbBr}_{3}$ nanomaterial in the presence of $\mathrm{EuBr}_{2}$ nanodots. The hybrid nanoplatelet exhibits the strong excitonic and narrow emission peaks characteristic of ultrathin NPLs at 430 and $436 \mathrm{~nm}$, respectively, and remains mainly non-stacked after prolonged standing in solution.

Lead halide perovskites are relevant semiconductor materials with the $\mathrm{APbX}_{3}$ formula, where $\mathrm{A}$ is a small-sized monocation (such as methylammonium, formamidinium, or cesium), and $X$ is a halide $\left(\mathrm{Cl}^{-}, \mathrm{Br}^{-}\right.$and/or $\left.\mathrm{I}^{-}\right)$. These materials present a threedimensional (3D) inorganic framework consisting of cornersharing $\mathrm{PbX}_{6}$ octahedra and the small-sized cations in the voids between them. They can easily be prepared with 3D morphology (bulk material) by simply mixing $\mathrm{AX}$ and $\mathrm{PbX}_{2}$, and also with other morphologies ${ }^{1}$ : nanoparticles (OD material) and low-dimensional materials (such as nanowires and nanoplatelets, 1D and 2D materials, respectively). Recently the preparation of colloidal nanoparticles has been reported with photoluminescence quantum yields ( $\Phi_{\mathrm{PL}}$ ) of up to $100 \%$, by using coordinating ligands which confine the material to the nanoscale and provide dispersibility; such nanoparticles are suitable for preparing thick films with a $\Phi_{\mathrm{PL}}$ close to that of the colloid. ${ }^{2-10}$ Their exceptional electronic and optical properties make them of great interest in light-emitting devices, photodetectors and photovoltaics. ${ }^{9,11,12}$

Currently, there is an increasing interest focused on perovskites with 2D morphology, in particular, nanoplatelets (NPLS) with a few lead halide layers and the $\mathrm{L}_{2}\left[\mathrm{APbX}_{3}\right]_{\mathrm{n}-1} \mathrm{PbX}_{4}$ formula, where " $L$ " represents the ligand and " $n$ " stands for the number of layers. ${ }^{13,14}$ As long as the NPL thickness is homogeneous, they exhibit the same emission peak, independently of their lateral dimensions, as well as exceptionally narrow absorption and emission (full width at half maximum (FWHM) ca. $10 \mathrm{~nm}$ ) and small Stokes shift $(<10 \mathrm{meV})$. They have been prepared as colloids following different strategies: i) exfoliation of the bulk material by sonication in the presence of coordinating solvents ${ }^{15}$; ii) hot-injection crystallization ${ }^{13}$; and iii) non-solvent crystallization ${ }^{16}$.

We devised that the ultrasound treatment of a mixture of two different materials could be used not only for the exfoliation of one of the materials, which might lead to a novel nanohybrid, but also for avoiding the stacking of the exfoliated material.
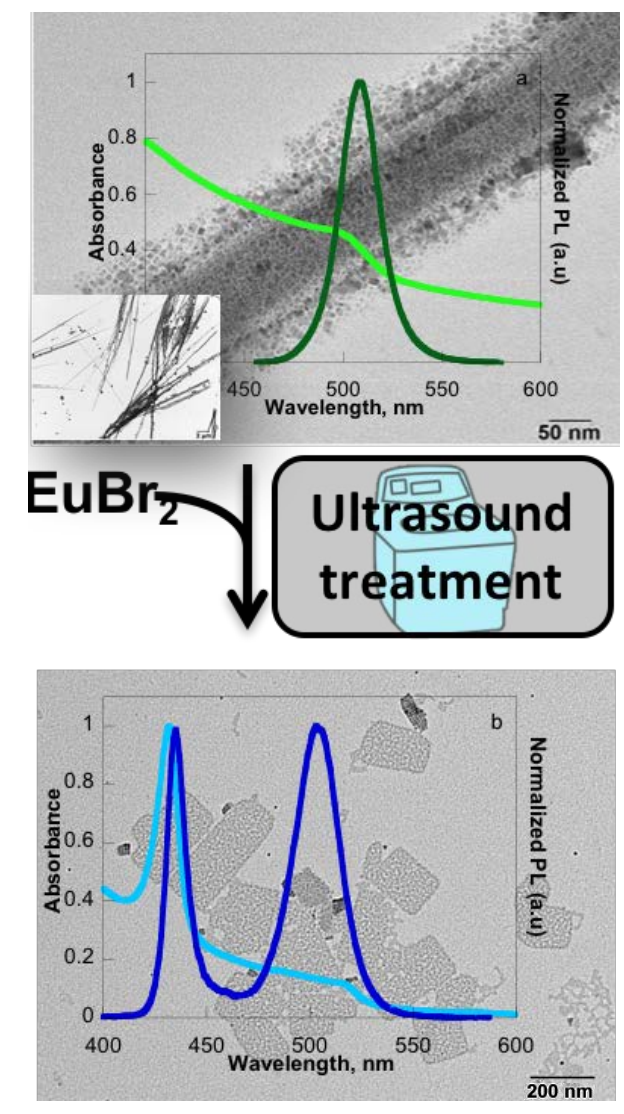

Figure 1: Transformation of $\mathrm{CsPbBr}_{3}$ nanoparticle nanowires (NPNWs) into ultrathin nanoplatelets (NPLs) under ultrasound/heating treatment in the presence of a $\mathrm{EuBr}_{2}$ solid. Top: conventional TEM image of the NPNWs (scale bar $50 \mathrm{~nm}$ ), absorption and emission spectra of the NPNWs; inset: TEM image at scale bar of $2 \mu \mathrm{m}$. Bottom: conventional TEM image of the NPLs (scale bar $200 \mathrm{~nm}$ ), absorption and emission spectra of the NPLs.

In particular, we report here that ultrasound/heating treatment of a toluene colloid of $\mathrm{CsPbBr}_{3}$ nanoparticles capped with dodecylamine (DDA) in the presence of europium(II) bromide nanodots $\left(\mathrm{EuBr}_{2} \mathrm{NDs}\right)$ lead to the formation of blue, highly stable two-layer $\left[\mathrm{CsPbBr}_{3}\right] \mathrm{PbBr}_{4}$ NPLs spotted with $\mathrm{EuBr}_{2}$ nanodots.

First, $\mathrm{CsPbBr}_{3}$ nanoparticles were prepared by the reprecipitation technique with some modifications. ${ }^{17,} 18$ Briefly, we prepared a solution containing an equimolar concentration of cesium bromide ( $\mathrm{CsBr})$ and lead bromide $\left(\mathrm{PbBr}_{2}\right)$ in a 9:1 dimethylformamide/dimethylsulfoxide mixture. Then, $200 \mu \mathrm{L}$ of this solution were mixed with DDA $(0.053 \mathrm{mmol})$ to obtain the precursor solution, which was added dropwise to a toluene solution of myristic acid (MA, $0.709 \mathrm{mmol}$ ); therefore, the DDA:MA molar ratio used in this preparation was 7:94 (see 
experimental section for further details). Finally, the dispersion was centrifuged at $5500 \mathrm{rpm}$ for $5 \mathrm{~min}$ and the precipitate was redispersed in $2 \mathrm{~mL}$ of toluene for further use and the supernatant was discarded. This combination of ligands led to the formation of micrometer-sized wires with a width of ca. 100 $\mathrm{nm}$, together with square-shaped nanoparticles of ca. $7 \mathrm{~nm}$ deposited at the edges of the wires; see Figure 1(top).

This material presented an exciton peak at $500 \mathrm{~nm}$, an emission maximum at $510 \mathrm{~nm}$ with a FWHM of $20 \mathrm{~nm}$ (Figure 1(top)), a $\Phi_{\mathrm{PL}}$ of $31 \%$ and an average PL lifetime $\left(\tau_{\mathrm{av}}\right)$ of $34.4 \mathrm{~ns}$ (the PL decay fitted to 3 exponential decays, see details in the experimental section); for further characterization data see thermogravimetry analysis (TGA), proton nuclear magnetic resonance (H-NMR), transmission electron microscopy (TEM), $\mathrm{X}$-ray photoelectron spectroscopy (XPS), and X-ray powder diffraction (PXRD) (Figure S1-S4).

In the TGA spectrum, the weight loss of $76 \%$ in the $100-200{ }^{\circ} \mathrm{C}$ range, assigned to the DDA ligand, was followed by a small loss (4\%) ascribed to MA, and then a loss of $20 \%$ in the $500-600{ }^{\circ} \mathrm{C}$ range attributed to the inorganic components $\left(\mathrm{PbBr}_{2}\right.$ and $\left.\mathrm{CsBr}\right)$ (Figure S1). The ${ }^{1} \mathrm{H}-\mathrm{NMR}$ spectrum corroborated that DDA was practically the only capping ligand on the $\mathrm{CsPbBr}_{3}$ NPNW's surface (Figure S2).

The XPS analysis of $\mathrm{Pb} 4 \mathrm{f}$ showed two symmetrical peaks at 138 and $143 \mathrm{eV}$ attributed to $\mathrm{Pb} 4 \mathrm{f}_{7 / 2}$ and $\mathrm{Pb} 4 \mathrm{f}_{5 / 2}$, respectively (Figure S3). The presence of two additional symmetrical bands at a lower energy, specifically at 137.7 and $142 \mathrm{eV}$, cannot be attributed to metallic lead or lead oxide ${ }^{19}$ and therefore, they could be indicative of perovskite $\mathrm{Pb}$ in a different environment. The peaks at 68.8 and $67.7 \mathrm{eV}$ were assigned to the $\mathrm{Br} 3 \mathrm{~d}$ present in the perovskite and are similar to those observed by other authors. ${ }^{20}$ The $\mathrm{N}$ 1s spectrum showed two peaks at 401.5 and $399.8 \mathrm{eV}$ that corresponded to DDA as ammonium and amine, respectively. ${ }^{21}$

The 01s spectra showed three peaks at 533.7, 532.4 and 531.3 $\mathrm{eV}$ attributed to the equivalent $\mathrm{O}$ atoms of the carboxylate, non-equivalent $\mathrm{O}$ of carboxylic acid, and to the O-O bond. The C 1s spectra exhibited an intense peak at $284.6 \mathrm{eV}$ assigned to the C-C aliphatic chain, which was used as a reference. Two small contributions at 288.3 and $286.7 \mathrm{eV}$ were ascribed to the carbonyl group and C-O bond of the MA and the peak at 285.4 eV corresponded to the $\mathrm{C}-\mathrm{N}$ signal. ${ }^{22}$ Finally, the typical peaks at 737 and $723 \mathrm{eV}$ were attributed to the Cs $3 \mathrm{~d} .{ }^{9}$

The powder XRD spectrum showed the presence of peaks at $15.08^{\circ}$ (002), 15.22 (110), $21.50^{\circ}(112), 30.38^{\circ}(004), 30.71^{0}$ (220) and $34.37^{\circ}$ (222); which are characteristic of the orthorhombic phase of $\mathrm{CsPbBr}_{3}$ (Figure S4). 23, 24

Secondly, the first attempt to prepare a hybrid material consisted of mixing a small amount of the freshly prepared $\mathrm{CsPbBr}_{3}$ colloid $(0.5 \mathrm{~mL})$ with the $\mathrm{EuBr}_{2}$ salt $(1.2 \mathrm{mg})$ in a vial and submitting the mixture to ultrasound/heating treatment. Small aliquots $(50 \mu \mathrm{L})$ were extracted after $1,3,5$ and $7 \mathrm{~h}$ and their UV-visible and PL spectra were systematically recorded (Figure S5). The absorption spectrum of the material after $7 \mathrm{~h}$ of treatment showed the appearance of a peak at $430 \mathrm{~nm}$ together with that of the excitonic peak of the $\mathrm{CsPbBr}_{3}$ perovskite at 500 $\mathrm{nm}$ (Figure SX). The PL spectrum showed two main peaks at 436 $\mathrm{nm}$ and $510 \mathrm{~nm}$; the first can be ascribed to the formation of two-layer caesium lead bromide nanoplatelets. Similar results were obtained when the treatment was performed for $7 \mathrm{~h}$ without sampling. Table S1 resumes the PL quantum yield of the reaction along the time.

The subsequent experiments were performed using $7 \mathrm{~h}$ of heating/ultrasonic treatment. Then, the synthesis was carried out using different amounts of: i) $\mathrm{EuBr}_{2}$ (0.6 mg, $1.6 \mathrm{mg}$ ), ii) $\mathrm{CsPbBr}_{3}$ colloid volume ( $\left.125 \mu \mathrm{L}, 250 \mu \mathrm{L}, 500 \mu \mathrm{L}\right)$, and iii) total volume of the mixture $(500 \mu \mathrm{L})$. The best results, in terms of dispersibility and emissive properties, were obtained using 0.6 $\mathrm{mg}$ of $\mathrm{EuBr}_{2}, 125 \mu \mathrm{L}$ of $\mathrm{CsPbBr}_{3}$ NPNWs and $500 \mu \mathrm{L}$ of the total volume. Figure 1 (bottom) shows the absorption and emission spectra that correspond to the colloid obtained after ultrasound/heating treatment of the $\mathrm{CsPbBr}_{3}$ NPNWs in the presence of $\mathrm{EuBr}_{2}$ for $7 \mathrm{~h}$. The PL $\left(\lambda_{\mathrm{ex}}=365 \mathrm{~nm}\right)$ spectrum of the optimal sample exhibited an emission at $436 \mathrm{~nm}$ and $510 \mathrm{~nm}$ with a total $\Phi_{\mathrm{PL}}$ of $41 \%$. The PL decays fitted to three components and the PL $\tau_{\mathrm{av}}$ was $16 \mathrm{~ns}$ and $8 \mathrm{~ns}$ for the emissions at $436 \mathrm{~nm}$ and $510 \mathrm{~nm}$, respectively. Table S2 contains the luminescence lifetime of the colloid obtained after ultrasound/heating treatment of $\mathrm{CsPbBr}_{3}$ NPNWs in the presence $\mathrm{EuBr}_{2}$ for $7 \mathrm{~h}$, recorded at $\lambda_{\text {ex }} 510 \mathrm{~nm}$ and $436 \mathrm{~nm}$. The

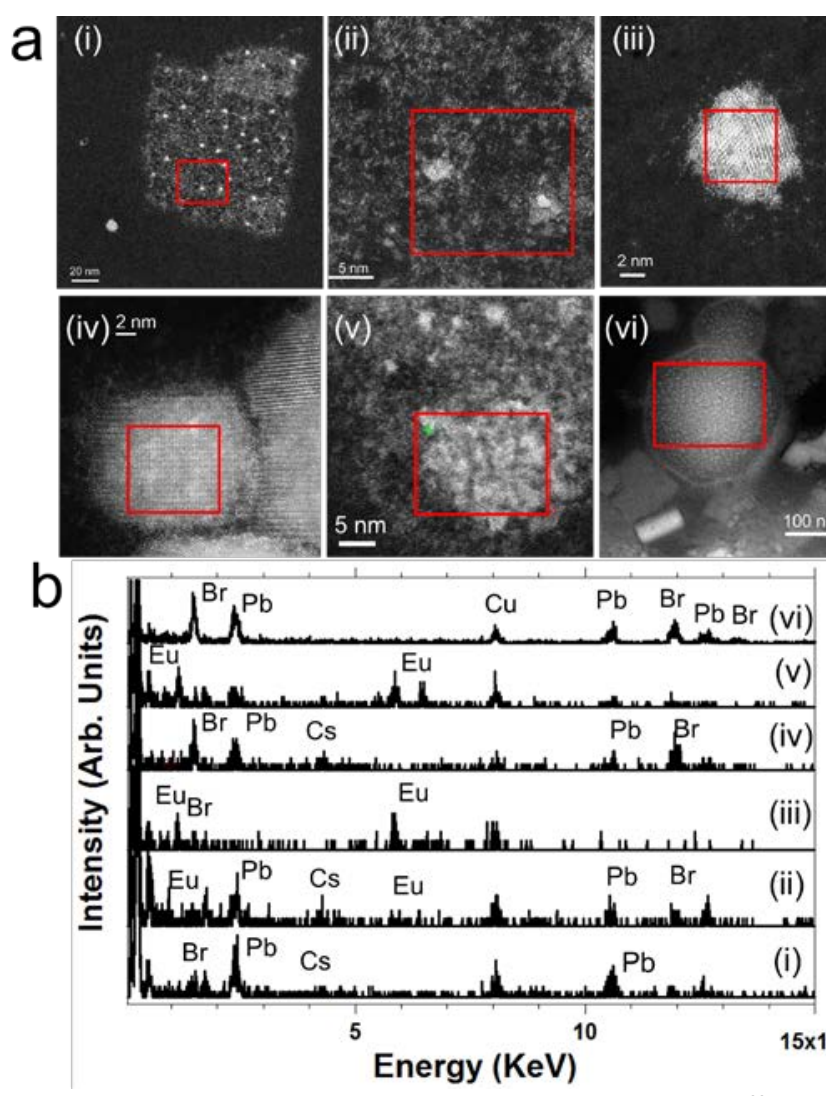

Figure 2: HAADF-HRSTEM images and EDS spectra recorded on different nanostructures present in the samples. (a) HAADF-STEM micrographs of: (i) a cesium lead bromide platelet, (ii) two $\mathrm{EuBr}_{2}$ nanodots, (iii) an $\mathrm{EuBr}_{2}$ nanoparticle, (iv) a perovskite NP, (v) an amorphous agglomerate containing europium and (vi) $\mathrm{PbBr}_{2} \mathrm{NP}$, respectively. (b) EDS spectra recorded rastering the electron beam on the highlighted red areas of each of the previous images. 
luminescence kinetics decays are shown in Figure S6. Furthermore, the relative intensity between the emission peaks did not change when exciting at $280 \mathrm{~nm}$, where $\mathrm{EuBr}_{2}$ does not absorb, thus ruling out the contribution of $\mathrm{EuBr}_{2}$ to the emission at $436 \mathrm{~nm} \cdot{ }^{25}$ Remarkably, the emission spectrum of the sample showed negligible changes after two weeks in air atmosphere (Figure S7).

Figure $\mathrm{S} 8$ shows the emission spectra $\left(\lambda_{\mathrm{ex}}\right.$ at $\left.365 \mathrm{~nm}\right)$ of the

a
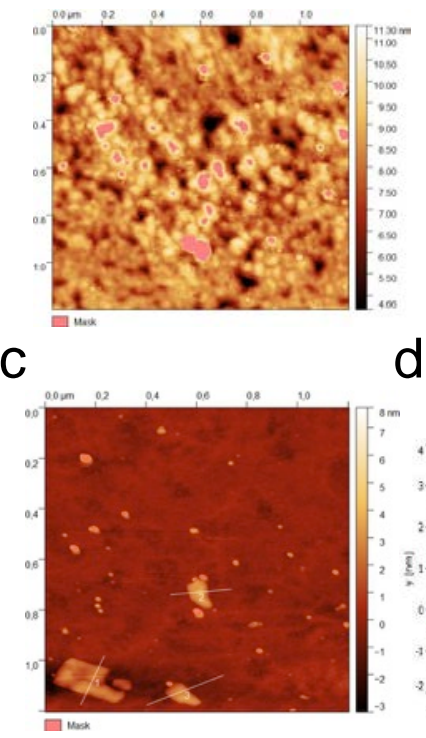

e

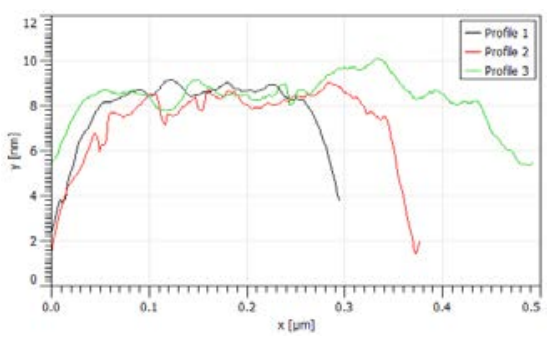

Figure 3: $a, b)$ Atomic force topography and height profile of the film prepared from the colloid obtained after sonication the $\mathrm{CsPBr}_{3} \mathrm{NPNWs}$ in the presence of $\mathrm{EuBr}_{2}$ (method 1). c,d) Atomic force topography, 3Dview and height profile of the film prepared from the colloid (method 2).

starting $\mathrm{CsPbBr}_{3}$ NPNWs in toluene and of the colloid obtained after ultrasound/heating treatment of the $\mathrm{CsPbBr}_{3}$ NPNWs in the presence $\mathrm{EuBr}_{2}$ for $7 \mathrm{~h}$, as well as the excitation spectra of the NPNWs $\left(\lambda_{\text {em }}\right.$ at $\left.510 \mathrm{~nm}\right)$ and of the colloid obtained after sonication of the $\mathrm{CsPbBr}_{3}$ NPNWs in the presence $\mathrm{EuBr}_{2}\left(\lambda_{\mathrm{em}}\right.$ at $515 \mathrm{~nm}$ and $430 \mathrm{~nm}$ ). All these data, together with the optical features reported for [ $\left.\mathrm{CsPbBr}_{3}\right] \mathrm{PbBr}_{4} \mathrm{NPLs}$, are consistent with the assignment of the emission at $436 \mathrm{~nm}$ to the NPLs.

Control experiments were carried out i) at a lower temperature and ii) in the absence of $\mathrm{EuBr}_{2}$. We found that the sonication bath at $70^{\circ} \mathrm{C}$ was crucial for the formation of the NPLs, since the corresponding excitonic peak was not detected when the reaction was carried out at $50{ }^{\circ} \mathrm{C}$ (Figure S9a, b). TEM images corroborated the nanoplatelets were not formed under this temperature (Figure S9c,d). This indicates that a suitable temperature during the ultrasound treatment is required. In addition, in the absence of $\mathrm{EuBr}_{2}$, the $\mathrm{CsPbBr}_{3}$ NPNWs transformed into micro-sized perovskite particles after $7 \mathrm{~h}$ of ultrasound/heating treatment. In keeping with this, the colour of the dispersion changed from yellow to orange and a drastic decrease in the sample dispersibility was observed. In addition, the excitonic peak at $500 \mathrm{~nm}$ shifted to $540 \mathrm{~nm}$, whereas the PL emission peak at $510 \mathrm{~nm}$ shifted to $550 \mathrm{~nm}$, and the PL decreased (Figure S10). TEM images in Table S3 show the different morphological evolution of the NPNWs in the presence than in the absence of $\mathrm{EuBr}_{2}$, under ultrasound/heating treatment. Figure S11 shows the HRTEM images of the colloid obtained after $7 \mathrm{~h}$, before and after centrifugation; it mainly consisted of $\left[\mathrm{CsPbBr}_{3}\right] \mathrm{PbBr}_{4} \mathrm{NPLs}$. The hypothesis is that sonication fragments the $\mathrm{CsPbBr}_{3}$ NPNWs as well as the $\mathrm{EuBr}_{2}$ solid and the europium salt helps in the exfoliation of the perovskite material.

The optimal precipitate was also analysed by high-angle annular dark-field (HAADF) scanning transmission electron microscopy (STEM) HAADF-HRSTEM combined with energydispersive X-ray spectroscopy (EDS) to determine its composition. ${ }^{26-28}$ These measurements, see Figure 2 , showed the formation of ultrathin cesium lead bromide platelets of different lateral sizes spotted with $\mathrm{EuBr}_{2}$ nanodots, homogeneously distributed throughout the platelet. In addition, the presence of $\mathrm{CsPbBr}_{3} \mathrm{NPs}, \mathrm{EuBr}_{2} \mathrm{NPs}$, and $\mathrm{PbBr}_{2}$ NPs, and amorphous material containing europium were clearly identified. A close inspection of the selected area electron diffraction (SAED) patterns of the $\left[\mathrm{CsPbBr}_{3}\right] \mathrm{PbBr}_{4}$ NPLs revealed the presence of orthorhombic $\mathrm{CsPbBr}_{3}$, preserving the main planes of the starting NPNWs. In addition, the plane (211), 4.21 of $\mathrm{EuBr}_{2}$ was detected in the sample, in agreement with the proposed NPLs spotted with $\mathrm{EuBr}_{2}$ (see Table S4 and Figure S12).

It is worth noting that europium vanished under prolonged irradiation of the NPL-EuBr${ }_{2}$ dots with the laser beam, while no evidence of damage of the perovskite layer was detected. Likewise, the $\mathrm{EuBr}_{2}$ nanodots were also damaged by electron beam. However, crystalline $\mathrm{EuBr}_{2} \mathrm{NPs}$, the $\mathrm{EuBr}_{2}$ covered with organic material and the $\mathrm{Eu}$ salts detected within the amorphous material remained stable under irradiation. Consequently, the EDAX analysis of NPLs-EuBr${ }_{2}$ dots cannot be used to determine the stoichiometry of these species.

AFM experiments (Figure 3 ) demonstrated that although we did not perform any further purification, deposition of a drop of the colloid on a glass substrate followed by spinning (method 1 ) led to homogeneous film, as demonstrated the roughness analysis of the AFM image and the 3D-AFM view (Figures 3a,b), with only few unfilled features, and the height profile gives a value of 8.6 $\mathrm{nm}$. This can be attributed to the tendency of the NPLs to lie flat on the substrate and stack together. Moreover, deposition of the colloid drop on a spinning glass (method 2) enabled the observation of some NPLs. Figures $3 c, d$ show the AFM topography and height profile with an average roughness of 3.1 $n m$ for the NPLs. 


\section{Conclusions}

In summary, we report here the preparation of a novel hybrid material consisting of a two-layer caesium lead bromide nanoplatelet with small $\mathrm{EuBr}_{2}$ dots homogeneously distributed in the structure. The strong excitonic and narrow emission peaks characteristic of ultrathin NPLs at 430 and $432 \mathrm{~nm}$, respectively, remained unchanged after two weeks under air atmosphere, thus showing no evolution towards more bulk-like structures. $\mathrm{EuBr}_{2}$ plays a key role in the formation of the perovskite nanoplatelet and the $\mathrm{EuBr}_{2}$ dots increase the chemical and colloidal stability of the platelets. The herein reported strategy opens the possibility to the development of hybrid materials with novel properties for application in photocatalysis and/or optoelectronics.

\section{Experimental section \\ Chemicals}

Cesium bromide (99.999\%, Sigma Aldrich, USA), lead bromide (99.999\%, Sigma Aldrich, USA), Europium(II) bromide (99.99\%, Alfa Aesar, Germany), Dodecylamine (98\%, Sigma Aldrich, USA), Myristic acid ( $\geq 99 \%$, Sigma Aldrich, USA), N,NDimethylformamide (99.8\%, VWR, USA), Dimethyl Sulfoxide ( $\geq 99 \%$, VWR, USA), Toluene anhydrous (99.8\%, Sigma Aldrich, USA).

\section{Characterization}

Absorption measurements. UV-vis spectra were recorded at room temperature using quartz cuvettes of $1 \mathrm{~cm} \times 1 \mathrm{~cm}$ using a UV-visible spectrophotometer Jasco V-670.

Luminescence measurements. Photoluminescence spectra were measured on an Aminco Browman series 2 Luminescence spectrometer, equipped with a xenon lamp (150 W). AB2 software (version 5.5) was used to register the data. Timeresolved $\mathrm{PL}$ were measured using a Compact fluorescence lifetime spectrometer C11367, Quantaurus-Tau. Fluorescence lifetime software U11487 was used to register the data. All of the data of PL decay of perovskite dispersed in ethyl acetate were acquired using $1 \mathrm{~cm} \times 1 \mathrm{~cm}$ path length quartz cuvettes and light-emitting diode excitation wavelength of $365 \mathrm{~nm}$. The $\mathrm{PL}$ decays of colloidal perovskite nanoparticles were fitted with a triexponential function. The average lifetimes $\left(\tau_{a v}\right)$ were calculated as $\tau_{a v}=\Sigma A_{i} \tau_{i}{ }^{2} / \Sigma A_{i} \tau_{i}$, where $\tau_{i}$ are the decay times and $\alpha i$ represents the amplitudes of the components, values obtained from the fitted PL kinetic decay traces.

\section{Transmission electron microscopy}

Transmission electron microscopy (TEM) was performed on a Tecnai G2 F20 microscope operated at $200 \mathrm{keV}$.

Quantum yield measurements. The photoluminescence (PL) quantum yields were measured using a Hamamatsu C9920-02 absolute PL quantum yield measurement system with monochromatic light source $(150 \mathrm{~W})$ and integrating sphere. All of the data were acquired using $1 \mathrm{~cm} \times 1 \mathrm{~cm}$ path length quartz cuvettes, at room temperature, using an excitation wavelength at $365 \mathrm{~nm}$.

Preparation of $\mathrm{CsPbBr}_{3}$ perovskite nanocrystals. The source of Cs used in this work was $\mathrm{CsBr}$, which was stored and manipulated in a glove box. After gridding of the salt, $8 \mathrm{mg}$ (0.037 $\mathrm{mmol})$ were dissolved in $1 \mathrm{~mL}$ of dimethylformamide

(DMF)/dimethyl sulfoxide (DMSO) mixture in a ratio 9:1, during 4 hours under ultrasound. The solution was transferred into a vial that contained $13.8 \mathrm{mg}$ of $\mathrm{PbBr}_{2}$ and the solution was called "precursor solution", with a molar ratio 1:1. An aliquot of this solution $(0.2 \mathrm{~mL})$ was mixed with $9.86 \mathrm{mg}(0.053 \mathrm{mmol}) \mathrm{de}$ dodecylamine (DDA). Finally, this mixture was injected into a toluene solution ( $2 \mathrm{~mL}$ ) of myristic acid (MA, $162 \mathrm{mg}$ ) and the $\mathrm{CsPbBr}_{3}$ nanocrystals were formed. The molar ratio used between the ligands was 7:94 for DDA:MA. The nanocrystals were purified by centrifugation steps ( $5500 \mathrm{rpm}$ for $5 \mathrm{~min}$ ) and the pellet redispersed in $2 \mathrm{~mL}$ of toluene.

Preparation of $\left[\mathrm{CsPbBr}_{3}\right] \mathrm{PbBr}_{4}$ NPLs. An aliquot of the $\mathrm{CsPbBr}_{3}$ nanocrystals $(125 \mu \mathrm{L})$ dispersed in toluene $(0.5 \mathrm{~mL})$ was mixed with 0.6 or $1.2 \mathrm{mg}$ of $\mathrm{EuBr}_{2}$ in a small vial. Then, the mixture was submitted to ultrasound/heating treatment for $7 \mathrm{~h}$ in a Branson 2510 Ultrasonic Cleaner. The final molar ratio between the components $\left(\mathrm{PbBr}_{2}: \mathrm{CsBr}_{\mathrm{EuBr}}\right)$ was 1:1:2 and 1:1:4 when using $0.6 \mathrm{mg}$ and $1.2 \mathrm{mg}$ of $\mathrm{EuBr}_{2}$, respectively. Finally, the nanomaterial formed was separated by centrifugation at 5500 $\mathrm{rpm}$ for $5 \mathrm{~min}$. The pellet obtained was redispersed in toluene $(0.5 \mathrm{~mL})$ to further characterization.

Scanning transmission electron microscopy (STEM) analyses. STEM (energy-dispersive X-ray spectroscopy (EDS) and highangle annular dark field (HAADF) STEM imaging) measurements were performed on probe-corrected FEI Titan Low-Base 60-300 operating at $300 \mathrm{keV}$ (fitted with a X-FEG ${ }^{\circledR}$ gun and Cs-probe corrector CESCOR from CEOS $\mathrm{GmbH}$ ). The convergent semiangle was of 25 mrad.

Atomic Force Microscopy. The AFM measurements were performed in a Nanoscope IVa Multimode Scanning Probe Microscope (Bruker, former Veeco); the microscope allows to measure surface characteristics as topography. The samples were prepared using $20 \mu \mathrm{L}$ of the colloid obtained after ultrasound/heating treatment of $\mathrm{CsPbBr}_{3}$ NPNWs in the presence $\mathrm{EuBr}_{2}$ for $7 \mathrm{~h}$.

\section{Conflicts of interest}

“There are no conflicts to declare”.

\section{Acknowledgements}

We thank MINECO (CTQ2017-82711-P and FPU for I.R-P), partially co-financed with FEDER funds; MAT2016-79776-P, Maria de Maeztu: MDM-2015-0538) for financing this research. RA also acknowledges Government of Aragon and ESF under the project "Construyendo Europa desde Aragon" 2014-2020 (grant number E13_17R). TEM measurements were performed in SCSIE (University of Valencia) or Laboratorio de Microscopias Avanzadas (Instituto de Nanociencia de Aragon-University of Zaragoza).

\section{Notes and references}


1. S. González-Carrero, R. E. Galian and J. Pérez-Prieto, Particle \& Particle Systems Characterization, 2015, 32, 709-720.

2. L. C. Schmidt, A. Pertegás, S. González-Carrero, O. Malinkiewicz, S. Agouram, G. Mínguez Espallargas, H. J. Bolink, R. E. Galian and J. Pérez-Prieto, Journal of the American Chemical Society, 2014, 136, 850-853.

3. S. Gonzalez-Carrero, L. Francés-Soriano, M. González-Béjar, S. Agouram, R. E. Galian and J. Pérez-Prieto, Small, 2016, 12, 5245-5250.

4. S. González-Carrero, L. Martínez-Sarti, M. Sessolo, R. E. Galian and J. Pérez-Prieto, Journal of Materials Chemistry C, 2018, 6, 6771-6777.

5. S. Gonzalez-Carrero, R. E. Galian and J. Pérez-Prieto, Opt. Express, 2016, 24, A285-A301.

6. F. Zhang, S. Huang, P. Wang, X. Chen, S. Zhao, Y. Dong and H. Zhong, Chemistry of Materials, 2017, 29, 3793-3799.

7. Q. Zhou, Z. Bai, W.-g. Lu, Y. Wang, B. Zou and H. Zhong, Advanced Materials, 2016, 28, 9163-9168.

8. H. Huang, M. I. Bodnarchuk, S. V. Kershaw, M. V. Kovalenko and A. L. Rogach, ACS Energy Letters, 2017, 2, 2071-2083.

9. X. Du, G. Wu, J. Cheng, H. Dang, K. Ma, Y.-W. Zhang, P.-F. Tan and S. Chen, RSC Advances, 2017, 7, 10391-10396.

10. L. Protesescu, S. Yakunin, M. I. Bodnarchuk, F. Krieg, R. Caputo, C. H. Hendon, R. X. Yang, A. Walsh and M. V. Kovalenko, Nano letters, 2015, 15, 3692-3696.

11. P. Ramasamy, D.-H. Lim, B. Kim, S.-H. Lee, M.-S. Lee and J.-S. Lee, Chemical Communications, 2016, 52, 2067-2070.

12. M. Kulbak, D. Cahen and G. Hodes, The Journal of Physical Chemistry Letters, 2015, 6, 2452-2456.

13. Y. Bekenstein, B. A. Koscher, S. W. Eaton, P. Yang and A. P. Alivisatos, Journal of the American Chemical Society, 2015, 137, 16008-16011.

14. J. Shamsi, Z. Dang, P. Bianchini, C. Canale, F. Di Stasio, R. Brescia, M. Prato and L. Manna, Journal of the American Chemical Society, 2016, 138, 7240-7243.

15. Y. Tong, E. Bladt, M. F. Aygüler, A. Manzi, K. Z. Milowska, V. A. Hintermayr, P. Docampo, S. Bals, A. S. Urban, L. Polavarapu and J. Feldmann, Angewandte Chemie International Edition, 2016, 55, 13887-13892.

16. M. C. Weidman, M. Seitz, S. D. Stranks and W. A. Tisdale, ACS Nano, 2016, 10, 7830-7839.

17. F. Zhang, H. Zhong, C. Chen, X.-g. Wu, X. Hu, H. Huang, J. Han, B. Zou and Y. Dong, ACS Nano, 2015, 9, 4533-4542.

18. S. Gonzalez-Carrero, L. C. Schmidt, I. Rosa-Pardo, L. MartínezSarti, M. Sessolo, R. E. Galian and J. Pérez-Prieto, ACS Omega, 2018, 3, 1298-1303.

19. M. Acik, I. K. Park, R. E. Koritala, G. Lee and R. A. Rosenberg, Journal of Materials Chemistry A, 2018, 6, 1423-1442.

20. S. Choudhury, S. Wei, Y. Ozhabes, D. Gunceler, M. J. Zachman, Z. Tu, J. H. Shin, P. Nath, A. Agrawal, L. F. Kourkoutis, T. A. Arias and L. A. Archer, Nature Communications, 2017, 8, 898.

21. S. Gonzalez-Carrero, R. E. Galian and J. Pérez-Prieto, Journal of Materials Chemistry A, 2015, 3, 9187-9193.

22. M. Lashkor, F. J. Rawson, J. A. Preece and P. M. Mendes, Analyst, 2014, 139, 5400-5408.

23. D. Zhang, S. W. Eaton, Y. Yu, L. Dou and P. Yang, Journal of the American Chemical Society, 2015, 137, 9230-9233.
24. D. Zhang, Y. Yang, Y. Bekenstein, Y. Yu, N. A. Gibson, A. B. Wong, S. W. Eaton, N. Kornienko, Q. Kong, M. Lai, A. P. Alivisatos, S. R. Leone and P. Yang, Journal of the American Chemical Society, 2016, 138, 7236-7239.

25. D. I. Galimov and R. G. Bulgakov, Luminescence, 2019, 34, 127 129.

26. C. Langlois, P. Benzo, R. Arenal, M. Benoit, J. Nicolai, N. Combe, A. Ponchet and M. J. Casanove, Nano letters, 2015, 15, 5075-5080.

27. R. Arenal, L. Henrard, L. Roiban, O. Ersen, J. Burgin and M. Treguer-Delapierre, The Journal of Physical Chemistry C, 2014, 118, 25643-25650.

28. M. Prieto, R. Arenal, L. Henrard, L. Gomez, V. Sebastian and M. Arruebo, The Journal of Physical Chemistry C, 2014, 118, 28804-28811. 